\title{
La atención de la escritura académica en el posgrado: una experiencia en la UPN
}

\author{
Ana Guadalupe Cruz Martínez \\ Universidad Pedagógica Nacional, Unidad 241
}

\section{Resumen}

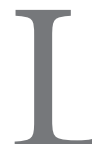

a escritura en el nivel de posgrado ha sido tema de preocupación en la Unidad 241 de la Universidad Pedagógica Nacional, en lugar de pre-ocuparse, se optó por ocuparse de la escritura a partir de la construcción de los textos con los que los estudiantes acreditan los diversos cursos y de la elaboración del proyecto de tesis. Lo que a continuación se presenta es la experiencia metodológica y los resultados del curso-taller Redacción de Textos Académicos y de Investigación (RETAI) que se llevó a cabo de forma presencial y en línea, tomando como base el principio discursivo del género académico con propósitos comunicativos. En este artículo se da cuenta del surgimiento del curso-taller, los contenidos que lo conforman, la metodología de evaluación entre pares que optimizó la revisión de textos propios y ajenos, así como de la prospectiva que dejó esta experiencia a nivel institucional.
Palabras clave: escritura en el posgrado, textos académicos, género académico, evaluación entre pares.

\section{Introducción}

La atención académica a la composición escrita en la Educación Superior en México, continúa como una asignatura pendiente. El tema de la escritura en el contexto docente e institucional no rebasa la frontera de pre-ocuparse, más que de ocuparse del problema. Existen esfuerzos on line en algunas instituciones nacionales como el Instituto Tecnológico de Estudios Superiores de Monterrey, la Universidad Nacional Autónoma de México, la Benemérita Universidad Autónoma de Puebla, la Universidad Popular Autónoma del Estado de Puebla y el Instituto Tecnológico Autónomo de México, sin embargo estas propuestas, que aunque son un buen apoyo, no dejan de ser herramientas remediales. 
Entre los docentes del nivel universitario, los comentarios sobre el déficit escritural con el que los estudiantes llegan a las aulas son recurrentes, en el caso particular del posgrado en la Unidad 241 de la Universidad Pedagógica Nacional (UPN en lo sucesivo) los alumnos "deben" desplegar estrategias de sobrevivencia para construir escritos ya que son éstos la única manera de acreditar determinados cursos.

Para el caso de América Latina, en países como Argentina, Venezuela, Chile y Colombia, la composición escrita y la redacción académica, son tareas que se encuentran incorporadas curricularmente a los diversos programas educativos. La composición escrita en la universidad es una responsabilidad de todos los docentes. En México, sigue siendo una tarea pendiente.

La preocupación por la escritura en la Educación Superior es uno de los temas emergentes; actualmente en la Universidad de Santa Bárbara, California, Charles Bazerman y su equipo de investigación, han puesto en marcha el proyecto Iniciativas de Lectura y Escritura en la Educación Superior en América Latina (ILEES) con el que se pretende delinear un mapa exhaustivo del desarrollo de la escritura en la Educación Superior en Latinoamérica a través de los sitios de práctica y las iniciativas de investigación.

La escritura en el nivel de posgrado requiere de grandes esfuerzos. En este nivel, el alumno ha de movilizar conocimientos, aprendizajes, habilidades y capacidades para la construcción de un texto académico. No solo son los saberes temáticos, también son aquellos sobre las formas escritas y la escritura de determinado texto.

La Unidad 241 de la UPN, no ha estado exenta de pre-ocuparse por el problema de la composición escrita en el posgrado; por varios años ha dado respuestas remediales y aisladas con talleres, cursos, algunos seminarios en los que el objeto de atención ha sido la escritura. Sin embargo, esto no ha resuelto el problema.

A partir del primer semestre del 2014, con el programa de Maestría en Desarrollo Educativo, línea Prácticas Curriculares de la Formación Docente, se inició en la institución un curso-taller de redacción académica permanente dirigido a estudiantes y egresados no titulados del posgrado. Lo que se presenta en este escrito es la experiencia vivida durante el semestre, así como de los avances obtenidos. La dinámica del curso-taller se sustentó bajo tres principios: 1. Género científico-académico, 2. Evaluación entre pares, y 3. Evaluación auténtica y aprendizaje centrado en el estudiante.

El propósito de este trabajo es el de compartir una iniciativa institucional para el abordaje de la redacción académica en el posgrado y la evaluación entre pares como estrategia para la revisión y retroalimentación de la redacción académica. Para fines documentales y de explicitación, el escrito se ha organizado en los siguientes apartados: problematización, justificación, objetivos, fundamentación teórica, metodología, resultados preliminares, conclusiones y referencias bibliográficas. 


\section{Problematización}

La composición escrita y la redacción académica son una necesidad sentida entre los estudiantes de la maestría. Los alumnos al concluir el primer semestre advierten la complejidad que les implica la elaboración de su proyecto de investigación, aducen que una dificultad es el desconocimiento metodológico para la construcción del proyecto, y otra, la gran pregunta: "qué voy a decir". Las manifestaciones de frustración, dicen, han sido muchas. El otro referente es la recuperación escrita que se hizo durante la aplicación de un diagnóstico, sobre las dificultades que encuentra el estudiante al empezar a escribir.

En el diagnóstico se detectaron dificultades temáticas, discursivas y lingüísticas. Dentro de las primeras, comentan su desconocimiento sobre el tema: "empíricamente lo he vivido" y la búsqueda de fuentes documentales: "además, nadie me dice dónde y cómo buscar". Las dificultades discursivas: "no sé qué tipo de escrito es, ni cómo estructurarlo", "la forma de ir articulando párrafos en un escrito", "cuál es el propósito del escrito", "no sé cómo argumentar y qué tipo de argumentos usar", "cómo conectar las ideas en los párrafos". Como problemas lingüísticos, donde los estudiantes presentan dificultades gramaticales como la acentuación, ortografía y puntuación. Un alumno comentó que "escribir me provoca angustia".

Los estudiantes de la maestría a través de la coordinación del programa, decidieron hacer la solicitud a la dirección de la Unidad 241 para que les dieran un curso de redacción. La dirección de la escuela apoyó la iniciativa de los alumnos y organizó el Curso Taller de Redacción de Textos Académicos y de Investigación (RETAI). Las preguntas que guiaron el proceso para su diseño fueron: ¿Cuáles son los conocimientos previos que poseen los estudiantes sobre la escritura académica? ¿Cómo identifican una redacción académica, de una que no lo es? ¿Cuáles son los conocimientos previos que poseen los estudiantes sobre la escritura especializada y científica? ¿Qué ubicación hace el estudiante de sí mismo ante el conocimiento de los diversos modelos de escritura? ¿Cómo planifica el estudiante sus textos? ¿Cómo evalúa un escrito?

\section{Justificación}

La redacción académica se enseña y se aprende escribiendo, revisando textos propios y ajenos. La redacción académica es una actividad institucionalizada que lejos de realizarse en solitario, debería formar parte de las actividades colaborativas de aprendizaje, máxime en la Educación Superior en la que la escritura se complejiza, ya que no solo se trata de evidenciar los conocimientos adquiridos en el análisis disciplinar, sino que con la escritura el estudiante transforma su conocimiento.

Los temas latentes durante la ejecución del curso-taller fueron escritura y evaluación (valoración) de la escritura. En cuanto a escritura fueron pertinentes los contenidos vinculados a modelos de escritura, tipología textual, género académico, textos especializados y científicos. La oportunidad de trabajar con estos contenidos, posibilitó que los estudiantes adquirieran elementos conceptuales y metodológicos para 
la redacción de los textos académicos más comúnmente solicitados en el posgrado, así mismo, sentaron las bases para la construcción de un texto científico.

La dinámica permanente para la evaluación-valoración de la escritura fue a través de pares. Esta manera de evaluar avizoró nuevas oportunidades de aprendizaje tanto para los alumnos como para la coordinación del taller. Lo aprendido pasó a formar parte de lo aprehendido. De una actitud insegura y frustración inicial, por parte de los estudiantes, a una actitud de cierta seguridad y respeto al otro. El curso-taller se convirtió en un espacio de aprendizaje académico valorado por los estudiantes de posgrado; después de su culminación se propone trabajarlo de manera permanente. Una de las metas es obtener la titulación del alumnado de la maestría, pero también que los estudiantes-egresados se inicien en la divulgación de sus saberes y de la investigación.

\section{Objetivo}

Esta experiencia pretendió proporcionar a los estudiantes de posgrado herramientas teóricas y metodológicas para la redacción de textos y géneros académicos y de aquellos que se originan como producto de la investigación, con la finalidad de consolidar la producción escrita en la construcción del texto científico, y con ello, incursionar en el ámbito de la divulgación del conocimiento.

\section{Fundamentación teórica}

Como ya se apuntaba en párrafos anteriores, los dos conceptos sobre los que se diseñó y llevó a cabo el curso taller fueron escritura y evaluación-valoración. De manera vinculatoria al concepto de escritura está el de textos, géneros académicos y discurso escrito. Al de evaluación-valoración, se halla la concepción de aprendizaje orientada al estudiante, a partir de los principios de la evaluación auténtica.

De acuerdo con Cassany (1999) la escritura es una manifestación lingüística intencionada y contextualizada; es un hecho social que se hace presente en un espacio y tiempo determinados con la venia de ser compartida por una comunidad específica. En este sentido, la escritura es funcional a partir de que se transforma en texto convencionalizado por un determinado código alfabético, pictórico, cronomático, etc. que cobra sentido en una determinada cultura. La escritura nace de una necesidad colectiva que la impulsa. Por lo tanto, "la escritura de un texto es un proceso situado y subsidiario de una determinada situación de comunicación" (Camps, 1995; Camps y Castelló, 1996; citados por Castelló, 2007: 49).

La escritura es la herramienta académica que da vida recursiva a los resultados de aprendizaje que obtienen los alumnos; tienen un efecto como actos de comunicación académica.

Escribir textos académicos es una actividad de construcción del texto propio, a partir de textos ajenos (intertextualidad), que da lugar a un producto final fruto del desarrollo de distintas posiciones enunciativas... producir textos académicos [es] una actividad que concibe el producto y la producción conjuntamente, en 
una perspectiva interactiva que asume el diálogo entre escritor y lector (Teberosky, 2007: 18).

El ámbito académico ha construido un género para dar a conocer lo que sucede en las aulas y en espacios disciplinares. Un discurso escrito en el que se moviliza la teoría, la exploración y la empiria. Es el discurso especializado, académico-científico.

Al respecto, Parodi (2005: 23) sostiene:

un modo de aproximación a los géneros especializados escritos en la academia, es partir desde el supuesto de que los materiales escritos empleados por los estudiantes en su formación universitaria cotidiana revelan tanto los tipos de textos como los rasgos representativos de su disciplina.

La evaluación del escrito académico en el posgrado cumple una función que va más allá de la acreditación de un curso, no se trata sólo de certificar saberes" (Carlino, 2012: 107) sino de proporcionar al estudiante "retroalimentación sobre su propio texto y la incorporación de las voces ajenas $\mathrm{y}$ de conocimientos ajenos (Beke, 2011: 132).

Para el desarrollo del curso-taller, la evaluación-valoración de la escritura se asume en contextos situados y orientada al aprendizaje del estudiante, a partir de algunos principios de la evaluación auténtica:

1. Explorar los aprendizajes que requieren habilidades cognitivas y acciones complejas, 2. Desarrollar tareas auténticas que representen tanto el contenido como las habilidades centrales, y de esta manera conjugar tanto la enseñanza como la evaluación, 3. Proporcionar a los alumnos los apoyos necesarios para realizar la actividad, 4. Comunicar con claridad las expectativas de ejecución en términos de criterios consensuados con el grupo, y 5. Incluir espacios de reflexión en torno a los aprendizajes logrados, a la enseñanza que los posibilitó y los mecanismos de evaluación que se emplearon (Vallejo y Molina, 2014: 18).

Para la evaluación de los trabajos escritos entre pares, se recuperaron los diez principios propuestos por Carlino (2012):

1. Reflexionar sobre el destinatario y el propósito del texto, 2. Dar a conocer los criterios de evaluación antes de ponerse a escribir, 3. Se enseña qué y cómo revisar, 4 . Ofertar tutorías para discutir borradores intermedios, 5. El docente retroalimenta la primera versión de lo escrito para posibilitar su reescritura optimizadora, 6. El docente da una valoración global, además de señalar problemas locales, 7. Hacer explícito el efecto de lo escrito sobre su destinatario, 8 . Se provee una escala de evaluación que detalla los rasgos de las producciones correspondientes a cada nota, 9. Los alumnos discuten y contribuyen a delinear los criterios de evaluación, y 10. Los alumnos, con guías del docente, leen y comentan las producciones de sus compañeros, para aprender a internalizar el punto de vista del lector, necesario para escribir en la academia (p. 112).

\section{Metodología}

El curso-taller desarrolló en sí mismo un proyecto de intervención. Durante el desarrollo del curso-taller, se pusieron en marcha supuestos de la investigación acción que plantea Stenhouse (1984) y Elliot 
(1993): los estudiantes aprenden investigando e indagando, con ideas guía, sobre su propia práctica y con aquellas que le son comunes; no se trata de conseguir resultados, sino de valorar el significado práctico de lo que se hace; cómo ser coherente entre la práctica y determinados principios pedagógicos; mejorar la calidad de la práctica misma.

Diseño y ejecución del curso-taller Redacción de Textos Académicos y de Investigación (RETAI): El curso-taller adoptó un carácter institucional. Fue avalado por la dirección de la Unidad 241 de la UPN. Estuvo dirigido a estudiantes de maestría y doctorado, así como a estudiantes egresados que aún no obtienen el grado. La invitación fue abierta; se publicó en la página web de la Unidad 241 de la UpN. Inició en marzo y concluyó en junio.

Se organizó en dos grandes temas: 1 . Redacción académica y 2. Géneros académicos. Para el primer tema se revisaron aspectos sobre la redacción académica y sus implicaciones lingüísticas y discursivas, los modelos de redacción y la revisión de experiencias exitosas en América Latina sobre las características de escritura en el posgrado. Durante el segundo tema se revisó una introducción a la teoría de los géneros académicos en el que se abordaron sus características según el propósito comunicativo; finalmente se trabajaron los textos académicos, especializados y científicos, las características que los distinguen y su construcción.

Se programaron 30 horas presenciales que estuvieron organizadas en 10 sesiones presenciales y 70 horas de trabajo indepen- diente en línea. Se conformó una plataforma (http//retai.upnslp.edu.mx) en moodle a la que los estudiantes podían recurrir libremente para la consulta de los materiales documentales de apoyo, revisar las actividades por sesión y subir comentarios o trabajos en el espacio de tareas.

La revisión inicial de los escritos fue sobre textos propios: la construcción del estado del arte, la justificación y la problematización del proyecto de tesis. Para el primero y segundo escrito se proporcionaron a los participantes insumos lingüísticos, discursivos y mínimos básicos de metodología de la investigación. Algunos insumos fueron la relación de marcadores textuales y su empleo, uso de elementos paralingüísticos en el escrito, la organización y estructura del párrafo así como información ejemplificada sobre los diferentes géneros de la escritura académica. También se realizaron ejercicios para identificar la actitud y el compromiso del autor con el texto, para ello, se recuperó el esquema propuesto por Bolivar (2014) sobre las categorías y subcategorías fundamentales en el uso de la modalidad en relación con el tipo de modalidad (deóntica o epistémica): modalidad, modalización, modulación, compromiso modal y atribución.

Para el tercer escrito, los indicadores fueron: enumeración de párrafos, signos de puntuación, marcadores textuales, oraciones principales y las formas argumentativas, estructura metodológica de la problematización del proyecto de tesis. Para cada uno de los indicadores existía un código de señalización que debía ser identificado en el escrito. 
Sujetos: Al curso-taller se inscribieron 18 estudiantes. De estos, 10 son estudiantes del 2do. semestre de la Maestría en Desarrollo Educativo, tres egresados de la Maestría en Educación, campo Formación Docente y Práctica Profesional de la subsede de Cedral, S.L.P. del Centro Regional de Educación Normal (CREN) y cinco egresados de otros programas de posgrado que oferta la Unidad 241.

Evaluación-valoración del escrito: La evaluación-valoración de los escritos se realizó de manera presencial. La dinámica fue entre pares bajo criterios e indicadores proporcionados por el coordinador del curso-taller. Las recomendaciones generales para la revisión de los textos fueron de carácter discursivo y lingüístico. Para ello, se les solicitó a los estudiantes elaboraran un cuadro comparativo a partir de la revisión de tres documentos; uno de Cassany La textura escrita (2010: 162-173) y dos de Serafini, Dudas lingüísticas y La puntuación (2009: 203-318).

El cuadro comparativo apoyó en mucho a los participantes, ya que les permitió una identificación conceptual de las características discursivas y de las lingüísticas. Con este ejercicio teórico conceptual se revisaron los escritos. Discursivamente se obtuvieron los propósitos comunicativos de los textos así como la ubicación del destinatario-lector virtual. Lingüísticamente se revisaron la coherencia y cohesión. Es decir el entramado de sentido entre los párrafos y la consistencia sintáctica (los aspectos lexicogramaticales y paralingüísticos). Los asistentes re-descubrieron y re-encontraron aprendizajes que ya poseían sobre el español como lengua materna.
Escritos especializados y de investigación: Para el análisis y revisión de los textos especializados y de investigación, se trabajó con modelos de textos de revistas especializadas en educación (Perfiles Educativos, Ries, Revista de Educación Superior, Revista Mexicana de Investigación Educativa) de las que se obtuvieron los criterios editoriales, las características tipográficas y de estructura de cada una de éstas. Y para la elaboración, se proporcionaron a los estudiantes documentos guía para la construcción de un ensayo, artículo científico, informe de investigación, reporte de investigación, reseña crítica y ponencia. Particularmente, para la revisión y construcción de la introducción de un artículo científico se trabajó con el modelo CARS (Create a Research Space) de J. Swales (1990). Este autor propone una estructura en la que se identifican los pasos retóricos a seguir para construir una introducción de un artículo científico: 1. Generalizaciones sobre el tema, 2. Descubrir "el nicho" o espacio vacío al que aportará el tema a tratar, y 3. Ocupar "el nicho", esto es la propuesta o intervención en ese espacio vacío del conocimiento sobre el tema.

\section{Resultados y prospectiva}

Los resultados que se presentan, son producto de la respuesta dada al propósito que orientó el diseño y ejecución del cursotaller y fundamentalmente de la opinión y productos que los propios estudiantes construyeron al término del curso-taller.

Cada uno de los estudiantes elaboró un artículo científico que será dictaminado por el comité editorial de la Revista Edu- 
cación y Sociedad, órgano de difusión de la Unidad 241 de la UPN. Para la mayoría de los estudiantes será la primera ocasión que aparecerá publicado uno de sus escritos.

La recuperación de la voz de los estudiantes en cuanto a las ventajas de contar con un curso-taller permanente de RETAI en la Unidad 241 de la UPN, se dio en dos momentos. En el primero se realizó un diagnóstico en el que se solicitó a los participantes manifestaran las principales dificultades que advierten al escribir; el segundo, al final, al recuperar los aprendizajes obtenidos durante el trayecto del cursotaller.

Al principio, algunas de las dificultades manifestadas: "la angustia de no saber cómo iniciar", "ausencia de palabras conectoras", "dificultad para parafrasear", "el conocimiento del tipo de texto que se va a escribir", "la estructura del tipo de texto", "la forma de ir articulando los párrafos en un escrito" "identificar cuál es el propósito del escrito", jerarquizar las ideas sobre lo que se quiere escribir", "conocer la estructura de un párrafo".

Algunos de los aprendizajes obtenidos: "la importancia de expresar las necesidades académicas. Cuando solicitamos apoyo para la redacción de nuestra tesis, no imaginábamos que se abriría una ventana tan grande... El curso-taller ha resultado uno de los mejores soportes en nuestro trayecto. Si bien es cierto que falta mucho por recorrer, ha permitido la percepción de esos 'pequeños detalles en la escritura'", "el apoyo entre pares. No sólo es dar cuenta de que, como se dice vulgarmente, 'se cojea del mismo pie', sino el hecho de que el curso-taller permitió encontrar, en los otros, miradas distintas a un mismo objeto", "reflexionar sobre la propia forma de redactar", "analizar los textos entre compañeros que resultaban desconocidos al inicio", "aprendí a leerme, a hacer una crítica de cómo escribo, las palabras que utilizo, a quién va dirigido, pero, sobre todo, a comunicarme desde mi postura en el trabajo de tesis que realizo".

Sobre la evaluación-valoración de los escritos dicen: "aprendí y me convencí de que todo escritor puede cometer errores. Esto me dio confianza para intentar redactar mi trabajo de tesis y mejorarlo", "el análisis que hacíamos de los textos me ayudó a llevarlo a cabo con mi tesis", "si antes procuraba tener cuidado al escribir, cuidando principalmente la puntuación y la ortografía, ahora, con conocimiento de causa reconozco también que se debe cuidar la forma en lo argumentativo y en la intención comunicativa", "las interacciones entre pares, permitió enfrentar la mirada del otro".

Otro de los resultados es la continuidad del curso-taller al interior del Seminario de Tesis II, en el que se trabajará con el proyecto de investigación de cada uno de los alumnos, bajo revisión entre pares, con productos escritos a metas concretas. Esto favorecerá la eficiencia terminal. Asimismo, acercar a esta actividad a los directores de tesis. Para el caso de los estudiantes egresados, se les hizo la invitación a seguir participando con el grupo que actualmente se encuentra cursando la maestría.

Esta experiencia se ha valorado institucionalmente, de tal suerte que a partir de septiembre se trabajará de manera inter- 
disciplinaria con los docentes adscritos al programa de Psicología Educativa, de reciente creación en la Unidad 241.

\section{Conclusiones}

Esta experiencia deja como alternativa la continuidad de un trabajo a mediano y largo plazo, sin embargo, no puede hacerse en solitario. Se requiere de la atención de los docentes que tienen a su cargo los diversos cursos del programa académico.

La evaluación-valoración entre pares resultó ser una opción didáctica pertinente para el aprendizaje de los estudiantes en cuanto a la composición escrita en el posgrado, pero se hace indispensable la opinión y valoración de los expertos en temáticas específicas. De esta manera, se seguirá el proceso de formación de los estudiantes para la evaluación entre pares, ya que no solo se revisará la escritura sino disciplinalmente qué dice el escrito.

Una de las tareas pendientes que deja este trabajo es la autoevaluación. Se contempla el inicio de un proceso reflexivo y autocrítico en el que se tome el escrito como objeto de evaluación. Es la escritura la que vehicula los conocimientos, habilidades y capacidades, incluso la actitud ética del estudiante. De esto debe dar cuenta la escritura.

\section{Bibliografía}

Beke, R. (2011). El conocimiento ajeno en las disciplinas. Referencias y citas. Lectura y Escritura para la Investigación. Caracas: Universidad Central de Venezue- la. Consejo de Desarrollo Científico y Humanístico.

Bolivar, A. (2014). Taller: Lectura y Escritura para la Investigación. Jornadas sobre lectura y escritura académica y científica. Cátedra UNESCO. Universidad Autónoma de Tlaxcala. Mayo 2014.

Carlino, P. (2008). Revisión entre pares en la formación de posgrado. Lectura y vida. Recuperado en http:// www.lecturayvida.fahce.unlp.edu.ar/numeros/ a29n2/2902Carlino.pdf

(2012). Escribir, leer y aprender en la universidad. Una introducción a la alfabetización académica. México: Fondo de Cultura Económica.

Cassany, D. (1999). Lo escrito desde el análisis del discurso. Lexis: Revista de lingüística y literatura, 23 ( 2). Recuperado en http://dialnet.unirioja.es/servlet/ articulo? codigo $=130537$

(2010). La cocina de la escritura. Barcelona: Anagrama.

Castelló, M. (2007). (Coord). El proceso de composición de textos académicos. Escribir y comunicarse en contextos científicos y académicos. pp 47-82. España: Graó.

Elliot, J. (1993). El cambio educativo desde la investigación-acción. Madrid: Morata.

Parodi S., G. (2005). Discurso especializado e instituciones formadoras: Encuentro entre lingüística $e$ informática. Discurso especializado e instituciones formadoras. Chile: Ediciones Universitarias de Valparaíso.

Proyecto IleEs: Hacia un mapa exhaustivo de la escritura en la educación terciaria en la región. Recuperado en http://www.ilees.org/

Serafini, M.A. (2009). Cómo se escribe. México: Paidós.

Stenhouse, L. (1984). Investigación y desarrollo del currículum. Madrid: Morata.

Swales, J. (1990) Research articles in English. Genre analysis. English in academic and research setting. 110-176. Cambridge: Cambridge University Press.

Teberosky, A. (2007). El texto académico. Escribir y comunicarse en contextos científicos y académicos. Conocimientos y estrategias. 17-46. España: Graó.

Vallejo R., M. y Molina S., J. (2014). La evaluación auténtica en los procesos educativos. Revista Iberoamericana de educación. 64. Recuperado en http://www. rieoei.org/rie64a01.pdf 